\title{
Platelet CD36 mediates interactions with endothelial cell-derived microparticles and contributes to thrombosis in mice
}

\author{
Arunima Ghosh, ${ }^{1,2}$ Wei Li, ${ }^{2}$ Maria Febbraio, ${ }^{2}$ Ricardo G. Espinola, ${ }^{3}$ Keith R. McCrae, ${ }^{3}$ \\ Erin Cockrell, ${ }^{4}$ and Roy L. Silverstein ${ }^{2}$ \\ 'Department of Biological, Geological, and Environmental Sciences, Cleveland State University, Cleveland, Ohio, USA. \\ 2Department of Cell Biology, Lerner Research Institute, Cleveland Clinic Foundation, Cleveland, Ohio, USA. 3Division of Hematology/Oncology and \\ ${ }^{4}$ Division of Pediatric Hematology, Case Western Reserve School of Medicine, Cleveland, Ohio, USA.
}

\begin{abstract}
CD36 is a scavenger receptor that binds multiple ligands, including phosphatidyl serine (PS). Although CD36mice do not have a bleeding diathesis, we show here that they do have significantly prolonged thrombotic occlusion times in response to $\mathrm{FeCl}_{3}$-induced vascular injury. Because cell-derived microparticles (MPs) are generated in response to vascular injury and circulate in patients with prothrombotic diseases, we hypothesized that PS exposed on their surfaces could be an endogenous CD36 ligand that transmits an activating signal to platelets. We found that MPs prepared from human ECs, monocytes, or platelets or isolated from blood of normal subjects bound to platelets. Binding was not observed with platelets from CD36- ${ }^{-}$donors and was inhibited by an anti-CD36 antibody or by blockade of exposed PS by annexin V or anti-PS IgM. Preincubation of platelets with MPs led to CD36-dependent augmentation of platelet activation in response to low doses of ADP, as assessed by measuring $\alpha_{2 b} \beta_{3}$ activation, P-selectin expression, and aggregation. Immunofluorescence confocal microscopy of murine carotid thrombi from $\mathrm{CD}^{-} 6^{-}$mice showed a significant decrement in endothelial antigen accumulation, which suggests that CD36 plays a role in MP recruitment into thrombi. These results provide what we believe to be a novel role for CD36 in thrombosis.
\end{abstract}

\section{Introduction}

CD36 is an 88,000-MW integral membrane protein expressed on platelets $(1,2)$, professional phagocytes $(3,4)$, microvascular endothelium (4), adipocytes (5), muscle cells (6), and certain specialized epithelium (6-8). It is a multifunctional receptor with independent capacity to bind at least 3 major classes of ligands: modified phospholipids, long-chain fatty acids, and thrombospondins. On ECs, CD36 mediates an antiangiogenic, proapoptotic response to thrombospondin-1 and $-2(9,10)$, while on fat and muscle cells, it functions to facilitate transport of long-chain fatty acids for storage or oxidative metabolism $(5,11)$. On phagocytic cells, such as macrophages, dendritic cells, microglia, and retinal pigment epithelia, CD36 is a scavenger receptor involved in recognition and uptake of oxidized LDLs (oxLDLs) (3,11-13), amyloid A $\beta$ peptide (14), apoptotic cells (15), shed photoreceptor outer segments (13), and certain microbial cell wall components (16). Numerous in vitro studies and experiments with $\mathrm{CD}^{-} 6^{-}$mice revealed a role for CD36 in a variety of important biological processes, including atherosclerosis (17), tumor angiogenesis (18), energy metabolism (19), inflammation (20), host defense $(21,22)$, antigen presentation (3), and taste (23).

Although CD36 was first isolated and structurally characterized from platelets (1), where it was termed glycoprotein IV or IIIb, its functional role on platelets has been obscure. CD36- mice have normal tail vein bleeding times and over many years of observation do not exhibit a bleeding diathesis. Human platelet CD36 defi-

Nonstandard abbreviations used: EMP, EC-derived MP; MMP, monocyte-derived MP; MP, microparticle; ox-, oxidized; PMP, platelet-derived MP; PPP, platelet-poor plasma; PRP, platelet-rich plasma; PS, phosphatidyl serine.

Conflict of interest: The authors have declared that no conflict of interest exists. Citation for this article: J. Clin. Invest. 118:1934-1943 (2008). doi:10.1172/JCI34904. ciency (known as the $\mathrm{Nak}^{\mathrm{a}-}$ phenotype) is common in Asian and African populations (24-27), and similar to the situation in mice, is not associated with an obvious bleeding diathesis. Recently our group, in collaboration with Podrez, Hazen, and Byzova, showed that atherogenic oxLDL binds and activates platelets in a CD36-dependent manner (28). The prothrombotic phenotype associated with hyperlipidemia in apoE-null mice fed a Westerntype high-fat diet was rescued by genetic deletion of CD36, demonstrating an important link among platelet CD36, hyperlipidemia, oxidant stress, and thrombosis. Because normal hemostasis is maintained in the absence of CD36, these studies suggest that CD36 might be an attractive target for antithrombotic therapy in the setting of atherosclerosis.

We now report a more general role for CD36 in thrombus formation in normal mice. This observation led us to hypothesize that CD36 ligands might be generated during vascular injury in the absence of hyperlipidemia and systemic oxidant stress. We were particularly interested in the role of microparticles (MPs) in this process, because previous studies from our lab and others demonstrated that CD36-dependent phagocyte recognition and uptake of apoptotic cells and/or shed photoreceptor outer segments was mediated by binding of CD36 to phosphatidyl serine (PS) and/or oxPS on their surfaces (13, 29-31). A characteristic feature of MP generation is the loss of membrane asymmetry, resulting in surface expression of PS. We thus hypothesized that PS on MPs might also act as a ligand for platelet CD36 and thereby promote platelet activation.

MPs are vesicular fragments that bud off normal cells during either activation or apoptosis $(32,33)$. They are typically $200-1,000$ $\mathrm{nm}$ in size and possess different antigenic properties depending on the type of cell from which they are derived. MPs are gener- 


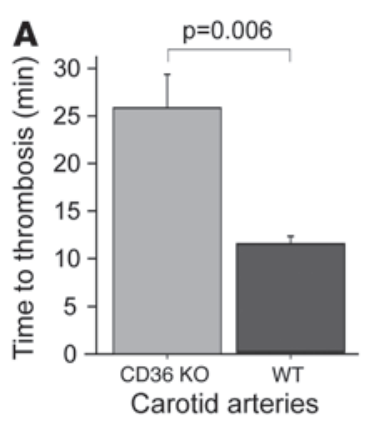

C
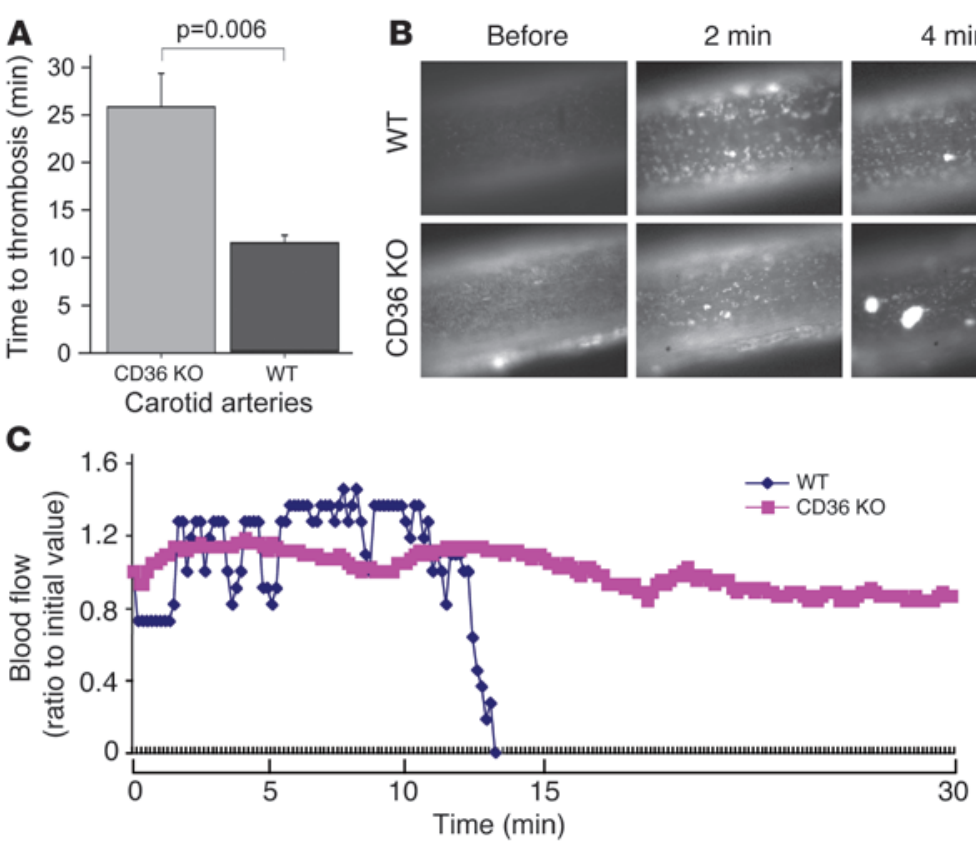
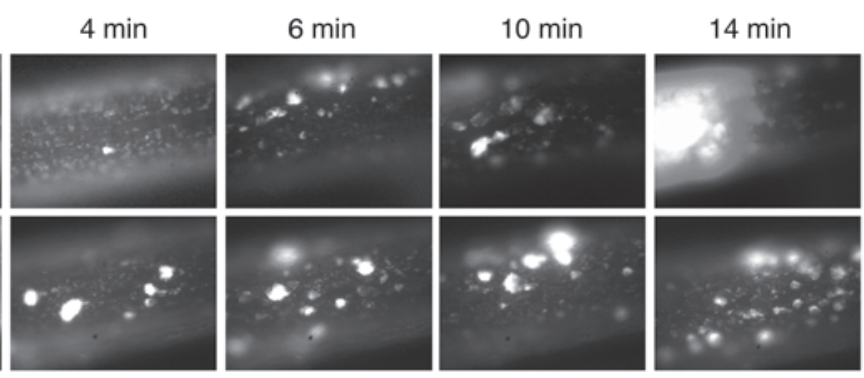

D

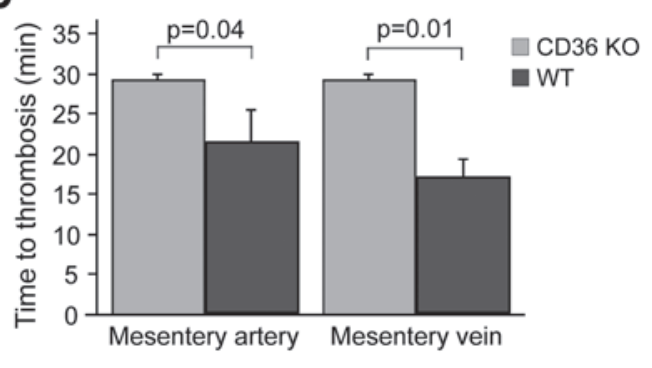

Figure 1

Increased thrombosis times in CD36- mice. (A) Time to thrombotic occlusion of carotid arteries from CD36- (KO) and matched WT mice was measured after 1-min topical application of $7.5 \% \mathrm{FeCl}_{3}$. Platelets were labeled by direct injection of fluorescence dye rhodamine $6 \mathrm{G}$ into the jugular vein, thrombi formation in the carotid artery was assessed under intravital microscopy, and blood flow was recorded with transonic flowmeter at the same time. $n=6$ per group. (B) Representative images of carotid arteries after injury induced by $7.5 \% \mathrm{FeCl}_{3}$. At 14 minutes, the artery from the CD36 WT mice was completely occluded, while the CD36- mice showed small thrombi with persistent blood flow. (C) Blood flow in the carotid arteries of WT and CD36- mice, expressed relative to the value before injury. (D) Time to thrombotic occlusion of mesenteric arterioles and venules was measured after application of $\mathrm{FeCl}_{3}$ as in $\mathbf{A} . n=10$ per group.

ated from platelets, monocytes, and ECs in the setting of vascular injury and were previously shown to become incorporated in developing thrombi in vivo (34). They have been postulated to play an important role in thrombosis, in part because PS on MP surfaces can be a site for catalytic assembly of the prothrombinase complex (35) and because monocyte-derived MPs are a rich source of tissue factor (36). EC-derived MPs (EMPs) can be generated by exposure to inflammatory cytokines and have been found in the blood of patients with thrombotic and inflammatory disorders, including acute coronary syndrome (37), sickle cell disease (38), diabetes mellitus $(39,40)$, thrombotic thrombocytopenic purpura (41), vasculitis, anti-phospholipid antibody syndrome (42), hypertension (43), and hematopoietic stem cell transplantation (44). In the present study we used MPs derived from cultured ECs, derived from purified human monocytes or platelets, or isolated from human subjects as model systems and showed that they bound to platelets in a CD36-dependent manner and sensitized them to activation by low doses of agonists. Furthermore, we showed that thrombi induced in $\mathrm{CD}^{3} 6^{-}$mice by topical $\mathrm{FeCl}_{3}$ application contained significantly fewer endothelial-derived antigens, supporting a role for CD36 in EMP incorporation into thrombi.

\section{Results}

CD36 deficiency prolongs in vivo time to thrombosis in murine models. We previously reported, using a carotid artery $\mathrm{FeCl}_{3}$ injury model, that thrombosis times in $\mathrm{CD}^{-} 6^{-}$mice were not significantly different from those in WT animals (28). Here we found that when the degree of vascular oxidative insult in this model was decreased by lower- ing the $\mathrm{FeCl}_{3}$ dose from $12.5 \%$ to $7.5 \%$, the time to complete thrombotic occlusion in $\mathrm{CD}^{-} 6^{-}$mice was more than doubled compared with that of WT animals (>25 versus 11 minutes; $P=0.01$; Figure $1, \mathrm{~A}$ and $\mathrm{B})$. Histologic and immunohistochemical vessel analysis comparing the 2 doses of $\mathrm{FeCl}_{3}$ did not reveal gross changes in elastic lamina disruption or endothelial or smooth muscle cell number at the light microscopy level, but hydroethidine staining revealed substantially less generation of reactive oxygen species after treatment with the lower dose (data not shown). The thrombosis times in WT mice exposed to the lower dose of $\mathrm{FeCl}_{3}$ were slightly longer than in those exposed to the $12.5 \%$ dose $(P=0.07)$, consistent with the hypothesis that the lower dose of $\mathrm{FeCl}_{3}$ was less prothrombotic. Visual imaging of thrombus formation in real time (Figure 1B) showed that thrombi formed more slowly in the null mice, but once formed, there did not appear to be any difference in clot stability in CD36- compared with WT mice. This was confirmed by simultaneous measurement of blood flow using a Doppler flow probe (Figure 1C). Similar results were seen in mesenteric arterioles and venules (Figure 1D), in which mean times to occlusion in $\mathrm{CD}^{-} 6^{-}$mice increased by $37 \%(P=0.04)$ and $68 \%$ $(P=0.01)$, respectively. These data show that CD36 plays a role in thrombosis in mice not subjected to hyperlipidemia and systemic oxidant stress and suggest that endogenous activating ligands for platelet CD36 may be generated during vascular injury.

MPs bind to platelets in a CD36- and PS-dependent manner. Because MPs are known to be generated in response to vascular injury, we reasoned that PS on the MP surface could serve as a CD36 ligand and contribute to platelet activation during thrombosis. As an 

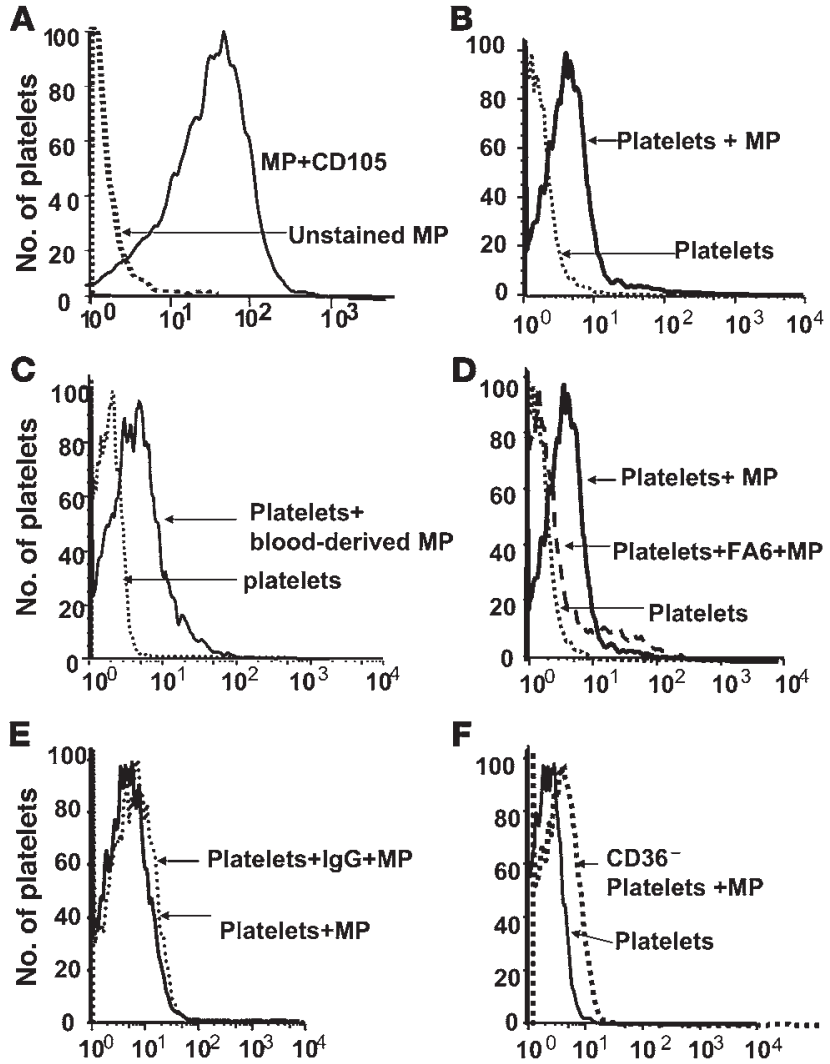

$=$

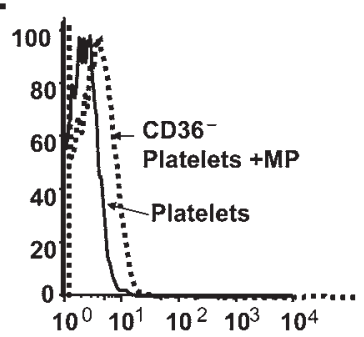

initial model system to test this hypothesis, we studied MPs generated from cultured early-passage HUVEC exposed to TNF and cycloheximide. As shown in Figure 2, EMPs, but not platelets, expressed CD105. When washed platelets were preincubated with EMPs prior to incubation with anti-CD105, the platelets acquired fluorescence (Figure 2B), which suggests that $\mathrm{CD} 105^{+}$EMPs were physically associated with the platelets. Titrating the ratio of MPs to platelets in this assay revealed that platelet-MP interactions were dose dependent and saturable, with a plateau of approximately 20 MPs per platelet. Similar results were seen using MPs generated from human peripheral blood monocytes (MMPs) by treatment with calcium ionophore and an anti-CD14 antibody as a detector (data not shown). The physical association of MPs with platelets was confirmed using 2-color fluorescence microscopy (Figure 3). EMPs were labeled with a red fluorophore, and platelets were labeled with a green fluorophore (Figure 3A). As shown in Figure $3 \mathrm{~B}$, the red EMP formed rosettes with the green platelets.

\section{Figure 3}

CD36-dependent binding of EMPs to platelets detected by immunofluorescence microscopy. (A) Top: Washed platelets were loaded with calcein and visualized by green fluorescence. Bottom: EMPs were loaded with PKH26 and visualized by red fluorescence. (B) PlateletEMP rosettes, seen when calcein-loaded platelets and PKH26-loaded EMPs were incubated together at a 1:9 ratio for $30 \mathrm{~min}$ prior to visualization. (C-F) Fluorescence-tagged platelets and EMPs were mixed together as in B, except that platelets were first incubated with (C) 50 $\mu \mathrm{g} / \mathrm{ml}$ oxLDL or (D) $50 \mu \mathrm{g} / \mathrm{ml}$ native LDL as control or (E) an inhibitory anti-CD36 IgG or (F) its IgG control prior to visualization. In all cases, images represent 1 of at least 3 separate experiments.

\section{Figure 2}

CD36-dependent binding of MPs to platelets, detected by immunofluorescence flow cytometry. (A) Flow cytometry demonstrated that EMPs generated and purified from HUVEC cultures treated with 100 $\mathrm{ng} / \mathrm{ml} \mathrm{TNF-} \alpha$ and $50 \mu \mathrm{g} / \mathrm{ml}$ cycloheximide for $24 \mathrm{~h}$ stained with a PEconjugated anti-CD105 lgG but not an isotype-matched control IgG. (B and C) Flow cytometry demonstrated that platelets did not react with the anti-CD105 lgG, but the platelets acquired PE fluorescence when incubated with (B) HUVEC-derived EMPs at a 9:1 EMP/platelet ratio or (C) blood-derived MPs isolated from a normal human subject at a 9:1 CD105+ MP/platelet ratio. (D and E) Platelets and HUVEC-derived EMPs were mixed and stained with anti-CD105 IgG as in B, except the platelets were first preincubated with (D) anti-CD36 IgG FA6 or (E) isotype-matched control IgG. (F) Platelets and HUVEC-derived EMPs were mixed together and stained with anti-CD105 IgG as in B, except platelets were from a CD36- donor. In all cases, histograms represent 1 of at least 5 separate experiments.

We used 2 independent methods to define the role of CD36 in platelet-EMP interactions. Preincubation of washed platelets with a monoclonal anti-CD36 antibody inhibited the acquisition of antiCD105 positivity in the flow cytometry assay (Figure 2D), whereas control IgG had no effect (Figure 2E). Similarly immunofluorescence microscopy demonstrated that oxLDL, an alternative ligand for CD36 (Figure 3C), and antibody to CD36 (Figure 3E) inhibited platelet-EMP interaction, whereas control IgG (Figure 3 F) and native LDL (Figure 3D) had no effect. Furthermore, platelets isolated from a CD36- donor did not bind EMPs (Figure 2F). We observed similar CD36-dependent physical association of MMPs or platelet-derived MPs (PMPs) with platelets (data not shown).

Next we sought to determine the role of PS on the MP surface in platelet binding. Incubation of EMPs with annexin $V$ to block exposed PS dramatically reduced platelet-acquired CD105 fluorescence (Figure 4). A control protein, thioredoxin, had no effect. In addition, incubation of MPs with a monoclonal anti-PS IgM antibody significantly reduced the platelet-MP interaction, whereas a nonimmune control IgM did not (Figure 4).

Many investigators have found that small numbers of MPs can be isolated from healthy human subjects with no thrombosis history or risk $(45,46)$. In order to demonstrate that the CD36-MP

A

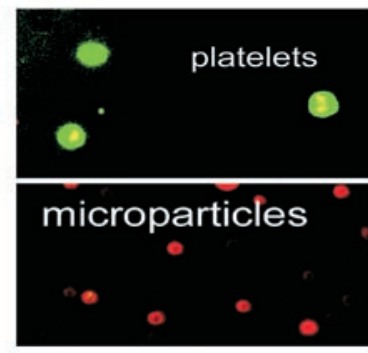

C

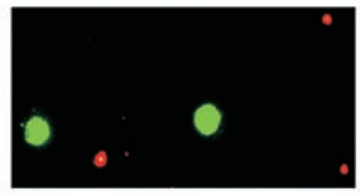

E

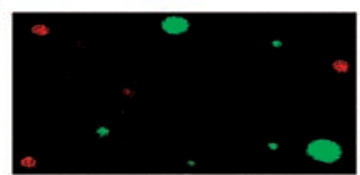

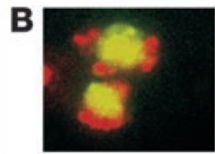
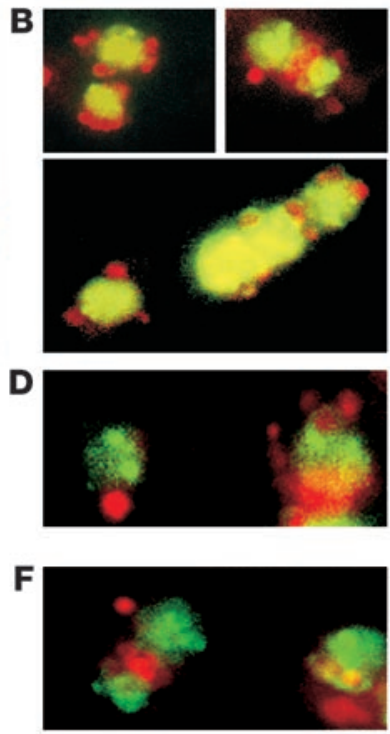
A
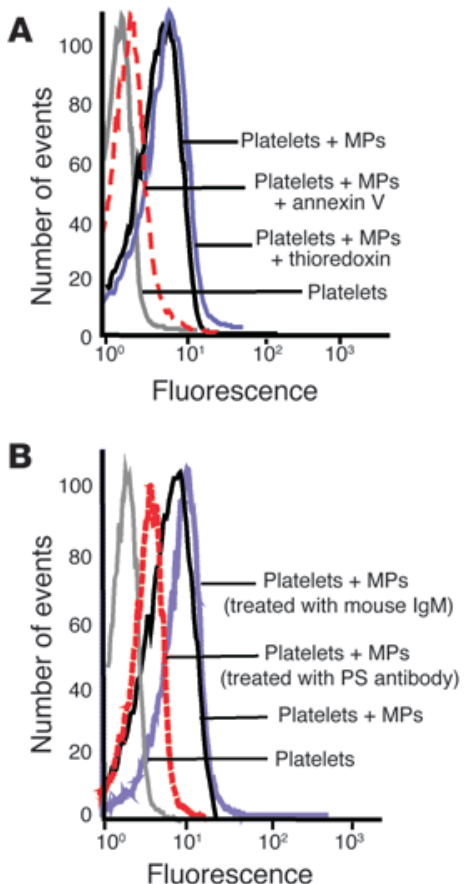

Fluorescence
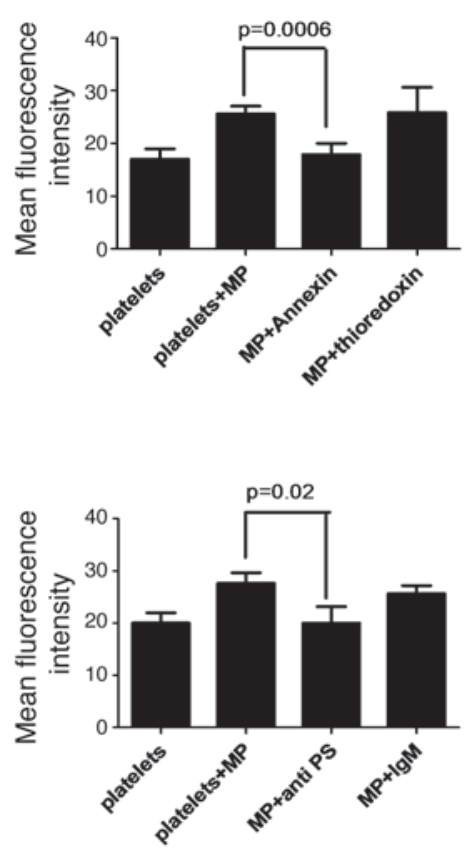

\section{Figure 4}

Binding of EMPs to platelets is PS dependent. Platelets and EMPs were mixed and stained with anti-CD105 IgG as in Figure 1B, except the EMPs were first preincubated with (A) $150 \mathrm{nM}$ annexin $\mathrm{V}$ or (B) $25 \mu \mathrm{g} / \mathrm{ml}$ monoclonal anti-PS IgM antibody to block exposed PS. Left: Flow cytometry from a representative single experiment. Right: Mean \pm SD data from at least 3 experiments. Plateletassociated anti-CD105 fluorescence was reduced by $>95 \%$ by annexin $\mathrm{V}$ and anti-PS IgM, while control proteins thioredoxin and nonimmune IgM had no effect. interactions described above were not caused by an artifact related to the in vitro culture systems used to generate MPs, we isolated and characterized MPs from the blood of 13 healthy human subjects. Table 1 shows that, as reported by others $(37,45,47,48)$, there was considerable variation in number and cellular origin among the donors. On average, approximately two-thirds of circulating MPs were platelet derived, with most of the remainder being endothelial, but in donors 1, 8, 9, and 10, the percent of EMPs was much higher. Figure $2 \mathrm{C}$ demonstrates that when washed platelets were preincubated with human blood-derived MPs at a 9:1 ratio of $\mathrm{CD} 105^{+}$MPs to platelets, the platelets acquired fluorescence, which suggests that $\mathrm{CD}_{105^{+}}$MPs were physically associated with the platelets. As with EMPs, the association of blood-derived MPs with platelets was inhibited by anti-CD36 antibodies and not seen with platelets from $\mathrm{CD}^{-} 6^{-}$donors (data not shown).

MPs enhance platelet activation and aggregation in a CD36-dependent manner. To assess the functional consequences of platelet-MP CD36 interactions, we incubated platelet-rich plasma (PRP) with MPs prior to assessment of activation and aggregation responses to graded concentrations of the agonists ADP and collagen. MPs alone did not induce platelet aggregation (data not shown), but in combination with low doses of ADP, EMPs at a 9:1 ratio of MPs to platelets induced a substantial increase in the rate and extent of the aggregation response (Figure 5A). This effect was not observed in platelets from CD36- donors (Figure 5B), demonstrating CD36 dependence. As with EMPs, we also found that preincubation of platelets with PMPs (Figure 5C) or MMPs (Figure 5E) augmented platelet aggregation responses to low-dose $\mathrm{ADP}$ with $\mathrm{CD} 36^{+}$platelets but not from platelets from a CD36- donor (Figure 5, D and F). Figure 5G shows that EMPs enhanced the extent of aggregation of $\mathrm{CD}^{2} 6^{+}$platelets induced by ADP concentrations of $1-4 \mu \mathrm{M}$ but not $20 \mu \mathrm{M}$. No EMP effect was observed at any ADP concentration in $\mathrm{CD}^{-} 6^{-}$platelets. Similar results were seen using collagen as an agonist; at concentrations of $4-10 \mu \mathrm{g} / \mathrm{ml}$ collagen, EMPs enhanced aggregation (data not shown).
To determine the effect of MPs on platelet activation, we also assessed expression of platelet P-selectin, a marker of $\alpha$-granule secretion (Figure 6A), and binding of PAC1, a marker of integrin $\alpha_{2 b} \beta_{3}$ activation (Figure $6 \mathrm{~B}$ ), in response to $2 \mu \mathrm{M}$ ADP. In both cases EMPs induced an increase in the expression of these markers. These effects were significantly diminished by preincubation of platelets with anti-CD36 IgG (Figure 6, A and B). Furthermore,

Table 1

Characteristics of MPs isolated from blood of normal human subjects

$\begin{array}{lcccc}\text { Donor } & \text { Annexin V APC } & \text { CD105 PE } & \text { CD14 PE } & \text { CD41 PE-Cy5 } \\ 1 & 44,730 & 6,030 & 1,980 & 900 \\ 2 & 180 & 270 & 90 & 450 \\ 3 & 8,190 & 3,960 & 1,125 & 73,440 \\ 4 & 9,450 & 3,458 & 225 & 23,940 \\ 5 & 9,630 & 2,655 & 720 & 2,250 \\ 6 & 13,950 & 1,485 & 585 & 1,680 \\ 7 & 360 & 180 & 135 & 2,295 \\ 8 & 5,040 & 4,500 & 315 & 2,520 \\ 9 & 540 & 3,420 & 135 & 1,710 \\ 10 & 5,490 & 2,025 & 90 & 1,350 \\ 11 & N A & 8,963 & 12 & 13,249 \\ 12 & 8,820 & 2,025 & 675 & 20,644 \\ 13 & 10,170 & 1,710 & 720 & 22,470\end{array}$

We analyzed 13 subjects and used MPs isolated from them for our experiments. Human MPs were isolated from PPP of normal human subjects, analyzed by flow cytometry, and quantified by light scatter and annexin $\mathrm{V}$ staining. MPs were also characterized by cell of origin using antibodies to endothelial (CD105), platelet (CD41), and monocytic (CD14) markers. Each marker was separately counted by flow cytometry; values are expressed in count per ml. Mean \pm SD for each MP marker was as follows: annexin V, 7,213 $\pm 4,988$ counts/ml; CD105, 3,238 $\pm 2,453$ counts/ml; CD14, $524 \pm 352$ counts/ml; CD41, $8,531 \pm 9,412$ counts/ml. NA, not available. 

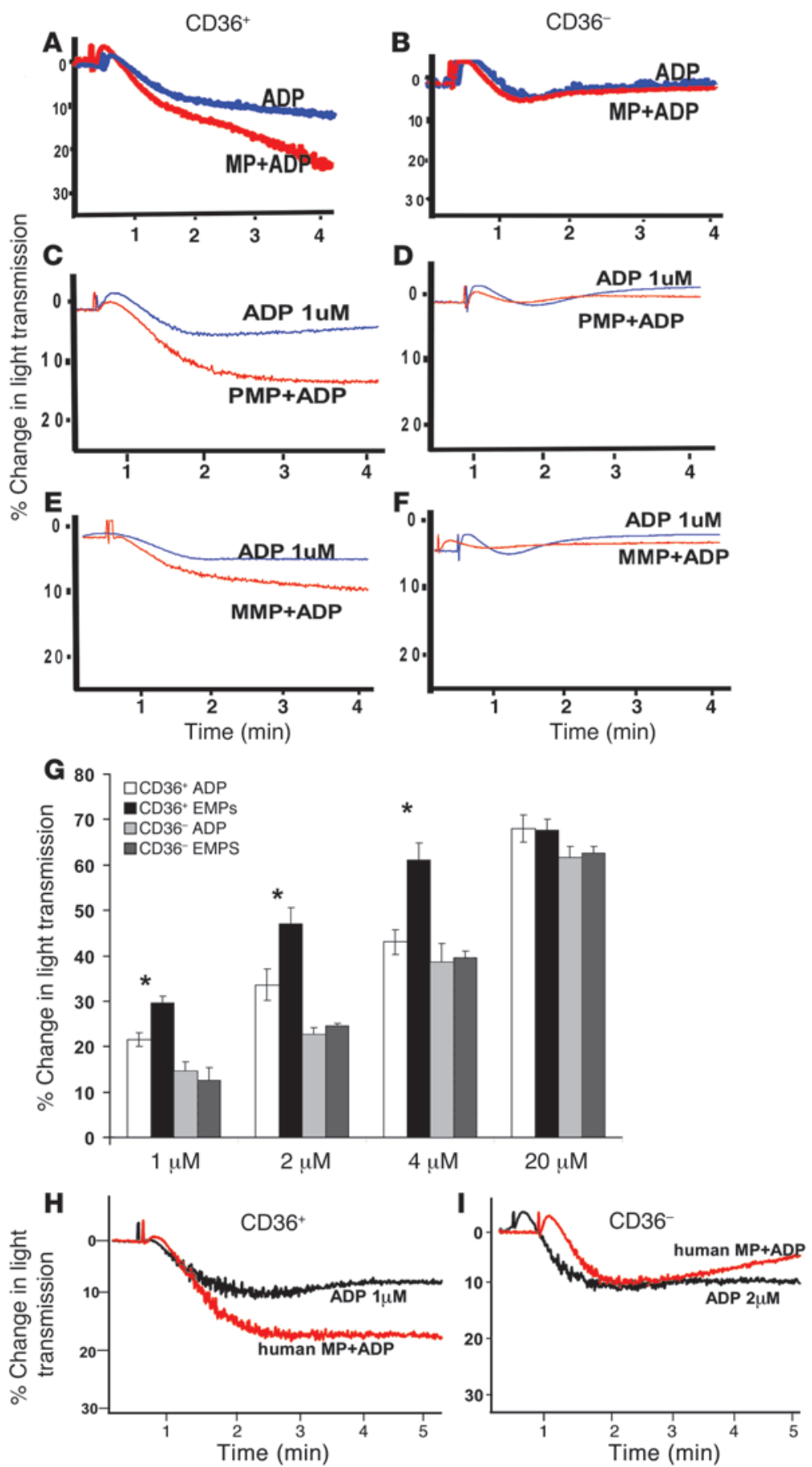

\section{Figure 5}

CD36-dependent enhancement of platelet aggregation by MPs in response to low doses of ADP. HUVECderived EMPs (A and $\mathbf{B}$ ) or human blood-derived MPs ( $\mathbf{H}$ and $\mathbf{I})$, MMPs (E and $\mathbf{F}$ ), or PMPs (C and $\mathbf{D})$ were added to PRP obtained from healthy $\mathrm{CD}^{+} 6^{+}(\mathbf{A}, \mathbf{C}, \mathbf{E}$, and $\mathbf{H})$ or CD36- $^{-}$(B, D, F, and I) donors and then stimulated with 1 or $2 \mu \mathrm{M}$ ADP as indicated. Aggregation was assessed turbidimetrically with a dual-channel aggregometer. Shown are representative aggregometry curves $(n=4)$. (G) Maximum aggregation response of $\mathrm{CD} 6^{+}$and CD36- platelets incubated with HUVEC-derived EMPs or with ADP alone, as indicated, in response to graded doses of ADP. Values are mean $\pm S D$ of measurements done in triplicate. ${ }^{*} P<0.01$.

with blood-derived MPs prior to exposure to $2 \mu \mathrm{M}$ ADP (Figure 6E). This effect was significantly diminished by preincubation of platelets with anti-CD36 IgG, showing CD36 dependence. These results demonstrate that blood-borne MPs affect platelet function in a manner similar to that of culture-derived MPs.

Expression of EC markers is reduced within carotid thrombi from $\mathrm{CD} 36^{-}$mice. Because murine $\mathrm{CD} 36$ deficiency prolonged in vivo time to thrombosis after $\mathrm{FeCl}_{3}$ injury, and because CD36 mediated a physical interaction between platelets and MPs, we next examined thrombi by immunofluorescence confocal microscopy to determine the relative degree of accumulation of the endothelial-specific antigen CD105. We reasoned that since CD105 is not known to be shed from the cell surface, any expression within a thrombus most likely reflects accumulated EMPs. Murine carotid thrombi expressed abundant CD105 antigen, which colocalized with the platelet marker CD61 (Figure 7A). Thrombi from CD36- mice expressed significantly less CD105 (62\% decrease in fluorescence intensity normalized to area of thrombus; $P=0.006$; Figure 7, $\mathrm{A}$ and $\mathrm{B})$, consistent with a decrement in EMP accumulation. The residual CD105 staining in thrombi from $\mathrm{CD}^{-} 6^{-}$mice suggests that CD36-PS binding may not be the only mechanism of platelet-MP interaction. It is highly likely during thrombus formation in vivo that MPs interact with activated platelets and leukocytes through additional mechanisms (e.g., P-selectin/PSGL1 and ICAM/MAC1), and perhaps with fibrin and other elements of the thrombus.

\section{Discussion}

Here we demonstrated, for the first time to our washed platelets from a CD36- donor showed neither increased P-selectin expression nor increased PAC1 binding in the presence of EMPs and $2 \mu \mathrm{M}$ ADP (Figure 6, C and D).

In order to show that the effect of MPs on platelet function was not limited to MPs generated from tissue culture or purified cellular systems, we isolated MPs from blood of normal human subjects and found that these also augmented platelet aggregation in response to low doses of ADP (Figure 5H). This was not observed when human blood-derived MPs were incubated with CD36- platelets (Figure 5I). PAC1 binding also increased when platelets were preincubated knowledge, that CD36 contributes to thrombus formation in response to vascular injury in vivo in mice maintained in standard vivarium conditions. The role for CD36 could not be demonstrated in the absence of predisposing conditions that lead to generation of CD36 ligands (e.g., hyperlipidemia and systemic oxidant stress) unless the degree of vascular oxidant insult was modulated by using a lower dose of $\mathrm{FeCl}_{3}$ than is typically used by most investigators. Thrombosis times, however, were still quite brisk at this dose in WT animals. We believe that the CD36 role in thrombosis is likely related to its expression on plate- 


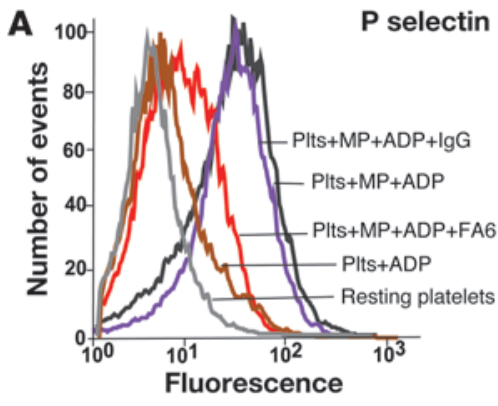

P selectin (CD36+)

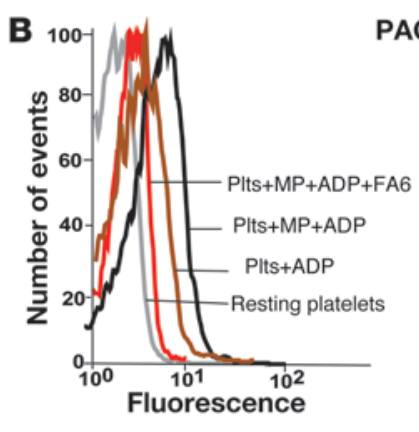

PAC1 (CD36+)
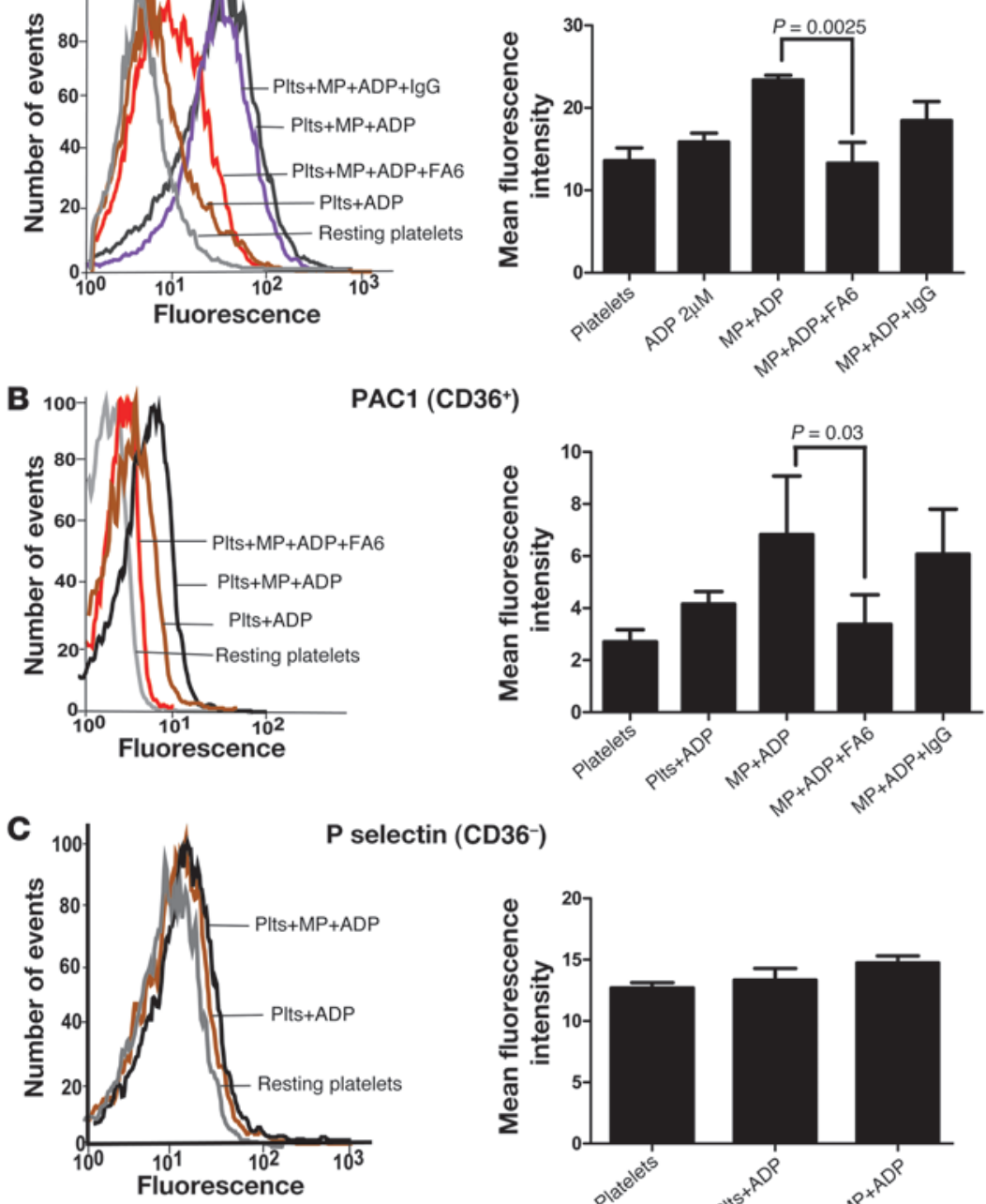

P selectin (CD36-)

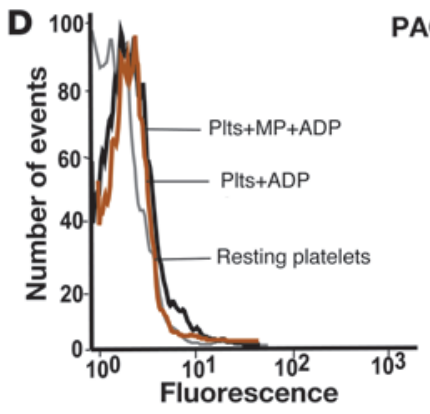

PAC1 (CD36-)
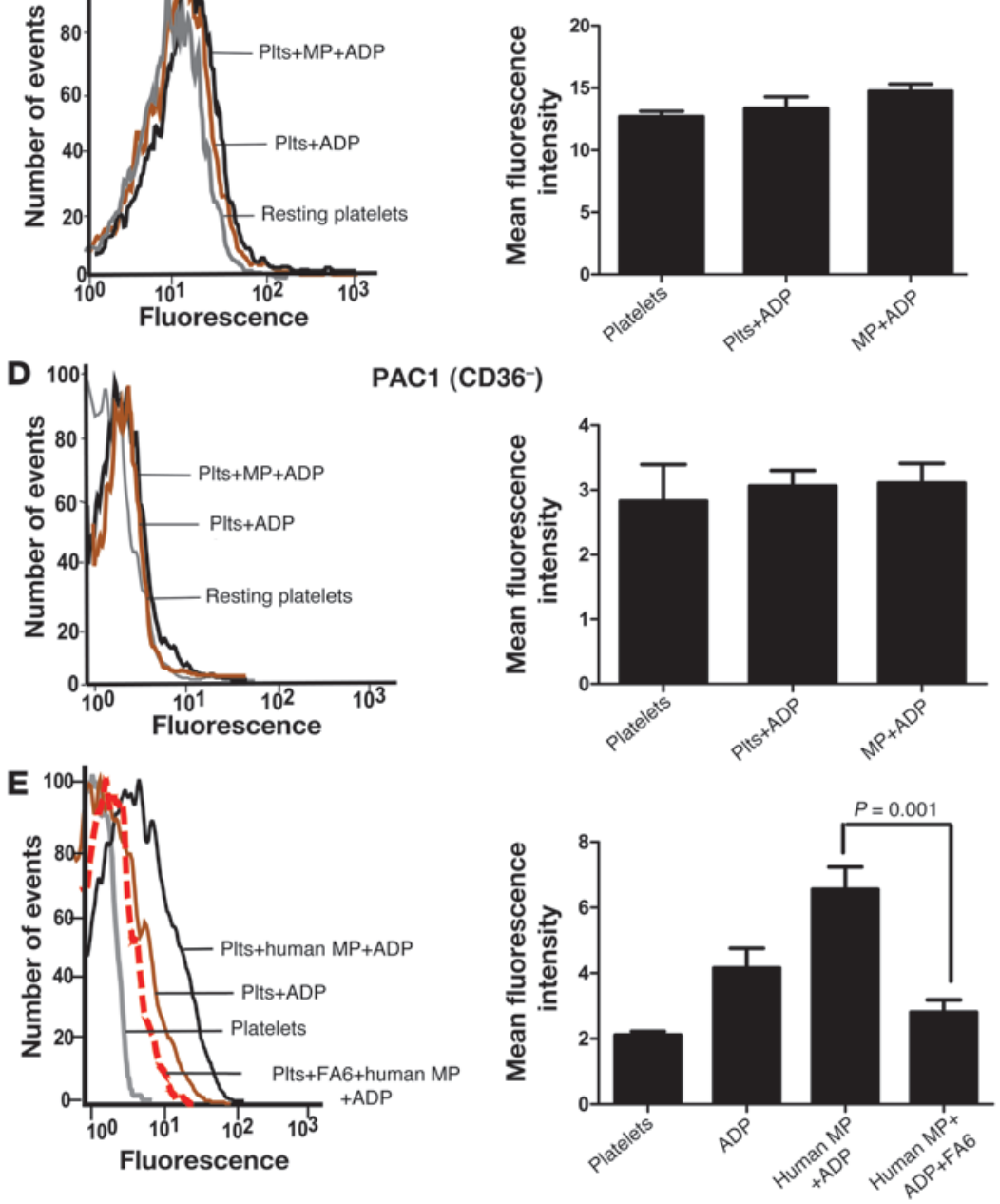

Figure 6

CD36-dependent enhancement of platelet activation by EMPs in response to low-dose ADP. Washed platelets from $\mathrm{CD} 36^{+}$donors (A, B, and E) or CD36- donors (C and $\mathbf{D})$ were incubated with HUVEC-derived EMPs at a 1:9 ratio or buffer control for $30 \mathrm{~min}$ and then stimulated with $2 \mu \mathrm{M}$ ADP. They were then incubated with either PE-conjugated anti-P-selectin (A and $\mathbf{C}$ ) or FITC-conjugated PAC1 (B, D, and E) and analyzed by flow cytometry. Anti-P-selectin and PAC1 bound to $\mathrm{CD} 36^{+}$platelets when they were preincubated with EMPs. Binding was significantly decreased when platelets were preincubated with anti-CD36 IgG, but not an isotype-matched control IgG. No increase in anti-P-selectin (C) or PAC1 (D) binding was seen using $\mathrm{CD}^{-} 6^{-}$platelets. (E) PAC1 binding to platelets increased when they were preincubated with human blood-derived MPs. Binding was significantly decreased when platelets were preincubated with anti-CD36 IgG. Histograms are representative of at least 5 different experiments and of 3 independent donors. Bar graphs show mean \pm SD from at least 3 separate experiments.

lets, because the $\mathrm{FeCl}_{3}$ injury models are largely platelet dependent and because large-vessel ECs do not express CD36. In our previous studies we used hyperlipidemic apoE-null mice and platelet transfusion strategies to show that plateletspecific CD36 engagement by oxLDL induced activating signals that contributed to thrombus formation (28). Our present results thus imply that CD36 ligands are generated during vascular injury and that signals induced by these ligands contribute to thrombus formation.

Our studies support the hypothesis that MPs may be one such class of CD36 ligand generated during vascular injury. Using inhibitory antibodies and competing ligands, as well as platelets from CD36- donors, we showed that MPs bound to resting platelets in a CD36-dependent manner; using annexin $\mathrm{V}$ or a monoclonal antibody to PS to mask surface PS, we showed that it is PS exposed on MP surfaces during their formation that serves as a ligand for platelet CD36. This is consistent with previous studies from our lab and others demonstrating that PS and/or oxPS serve as ligands on apoptotic cells and shed photoreceptor outer segments facilitating CD36-dependent recognition and internalization by phagocytic cells $(13,29$, 30). These data also provide an explanation for our finding that MPs generated from multiple cellular sources interacted with platelet CD36, because loss of membrane asymmetry and PS exposure is a common feature of MP generation from all cells.

In previous studies, CD36 was shown to initiate a signal in phagocytes that resulted in internalization of bound ligands, such as oxLDL and apoptotic cells $(11,13-16)$. We and others have also defined CD36-mediated signals in macrophages that are required for oxLDL uptake and 

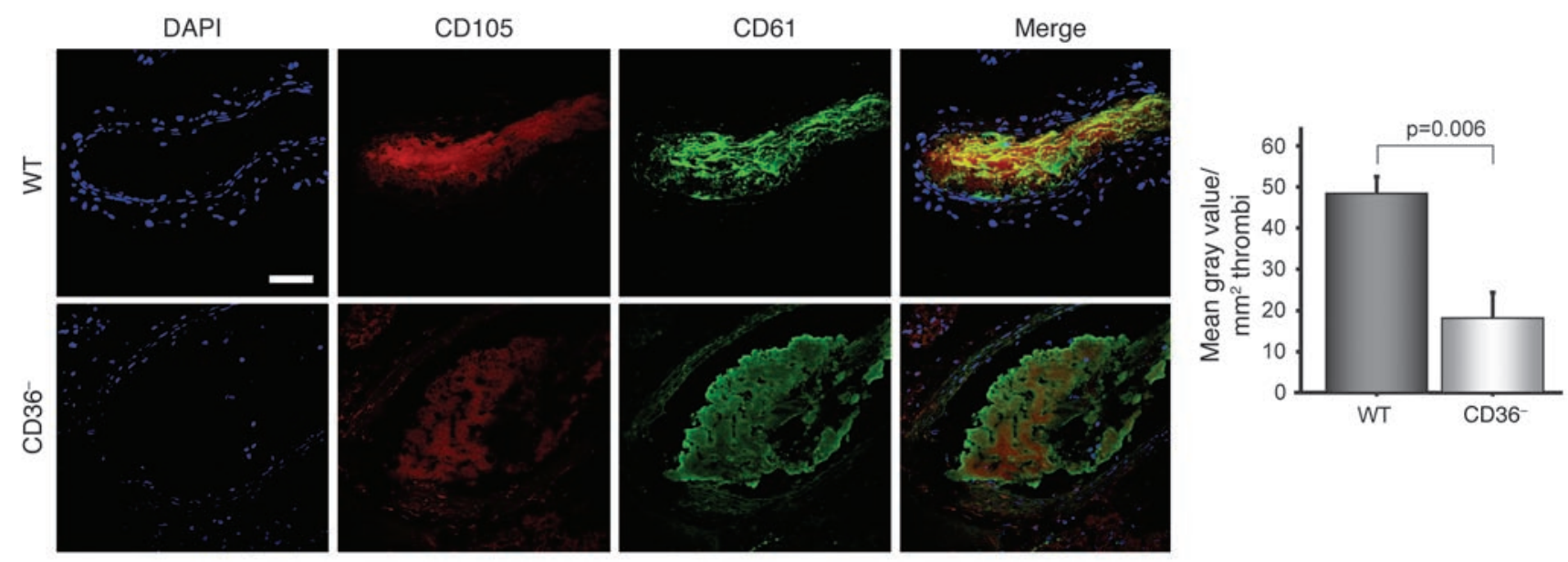

\section{Figure 7}

Accumulation of the endothelial-specific antigen CD105 is diminished in carotid thrombi from CD36- mice. Carotid arteries were removed from WT and CD36- mice after induction of occlusive thrombi with $\mathrm{FeCl}_{3}$ as described in Figure 1. Arteries were embedded in OCT and cross sectioned through the proximal to distal end of the thrombi. Sections from the middle portion of thrombi were double immunofluorescence stained with antibodies to endothelial-specific antigen CD105 (red) and platelet-specific antigen CD61 (green), and nuclei were stained with DAPI (blue). Original magnification, $\times 400$. Scale bar: $50 \mu \mathrm{m}$. Bar graph shows mean fluorescence intensity of CD105 staining. $n=4$ per group.

foam cell formation (49) and for clearance of bacterial products (16). Because platelets are probably not phagocytic cells, we hypothesized that CD36 signaling induced by MPs might instead modulate platelet activation. To test this hypothesis, we demonstrated using both turbidimetric platelet aggregation studies and flow cytometry assays that MPs significantly augmented platelet activation at low doses of the weak agonist ADP $(1-4 \mu \mathrm{M})$. This effect was abrogated by CD36-inhibitory antibodies and was not observed in platelets from CD36- donors.

The classic platelet activation model suggests that agonists, such as collagen, thrombin, and epinephrine, interact with platelet surface receptors, most of which are $G$ protein coupled, to initiate intracellular signaling events that lead to activation of integrins, reversible platelet aggregation, and secretion of platelet granule contents. Outside-in signals mediated by integrin $\alpha_{2 b} \beta_{3}$, and additional $G$ protein-coupled receptor activation via secretion of ADP and thromboxane, result in enhancement of the signal and ultimately stable platelet aggregation and thrombus formation. Recent studies have significantly advanced and refined this model. A large number of signaling molecules have been identified - both receptors and ligands - that act within the platelet-platelet contact zone after the aggregation event. These include ephrins and their receptors, eph kinases $(50,51)$; gas 6 and its tyrosine kinase receptors, mer, tyro3, and axl (52, 53); PECAM-1 (54); CD40 and CD40L $(55,56)$; and semaphorin 4D (57). It has been suggested that after aggregation, platelets form a synapse facilitating signaling by membrane-tethered receptor/ligand pairs and localizing secreted and shed ligands $(58,59)$. This promotes growth and stability of the thrombus.

Unlike most of the receptor/ligand pairs described above, CD36 may function on resting platelets to signal and sensitize platelets to activation by other agonists. Under conditions of low-grade vascular injury, as modeled in our in vivo system by topical application of $7.5 \% \mathrm{FeCl}_{3}$ and in our in vitro system by low-dose ADP, the CD36 signals became necessary for normal thrombus formation and full platelet activation. Immunofluorescence of thrombi from CD36 and WT mice in our studies were consistent with a role for platelet CD36 in recruitment of MPs into a developing thrombus. This could have an important effect on thrombus propagation, related both to direct effects of MPs on platelet function and to their ability to facilitate thrombin generation by presenting tissue factor and serving as an assembly site for the prothrombinase complex.

Importantly, the ligands that we have shown to bind CD36 on platelets (oxidized lipids and MPs) are generated endogenously in markedly increased amounts as a consequence of vascular injury and/or diseases known to be associated with increased thrombotic risk. We thus suggest that under these conditions, CD36 signals render platelets hyperreactive and may predispose patients to pathological thrombosis. Therefore, CD36 might be an attractive target for antithrombotic therapy, especially because no bleeding diathesis has been observed in humans or rodents with CD36 deficiency.

Our model does not preclude a role for CD36 in the platelet synapse. It is possible that ligands such as PS that are generated on the platelet surface during activation could interact with CD36 on neighboring platelets in the contact zone to increase responses to other signals and thereby provide a positive feedback loop during normal hemostasis. In fact we observed small, but significant, decrements in CD36- platelet responses to ADP (Figure 5G) and collagen (data not shown) in our studies, manifest as a left shift in the doseresponse curves. These shifts could be the result of loss of ability of CD36- platelets to respond to platelet-generated CD36 ligands.

\section{Methods}

Murine thrombosis models. All animal experiments were approved by the IACUC of the Cleveland Clinic. CD36- mice backcrossed 8 generations into a C57BL/ 6 background were obtained as previously described (17). Male CD36- mice of 22-26 g body weight and matched WT controls were anesthetized with ketamine $(90 \mathrm{mg} / \mathrm{kg})$ and xylazine $(15 \mathrm{mg} / \mathrm{kg})$, and the right jugular veins and the left carotid arteries were exposed via a midline cervical incision. Rhodamine $6 \mathrm{G}(0.5 \mathrm{mg} / \mathrm{ml} ; 100 \mu \mathrm{l})$ was injected directly into the right jugular vein to label platelets. The left common carotid artery 
was stripped of adventitia, a transonic flow probe (MA0.5PSB; Transonic Systems Inc.) was placed at the distal carotid artery, and a piece of black plastic was placed under the proximal vessel to reduce the background fluorescence. A $1 \times 2 \mathrm{~mm}$ piece of filter paper saturated with $7.5 \%$ or $12.5 \%$ $\mathrm{FeCl}_{3}$ solution was then applied to the proximal carotid artery for $1 \mathrm{~min}$, at which point the vessel was rinsed with saline and thrombus formation was observed in real time under a Leica DMLFS fluorescent microscope with an attached Gibraltar Platform (EXFO) and a water-immersion objective at $\times 10$ magnification. Blood flow was recorded at the same time. Time to thrombosis was determined through visual inspection using real-time video image capture with a QImaging Retigo Exi 12-bit mono digital camera and Streampix version 3.17.2 software (Norpix Inc.). The end points were set as either cessation of blood flow for $>30 \mathrm{~s}$ or no occlusion after $30 \mathrm{~min}$, in which case the time was recorded as $30 \mathrm{~min}$. In another set of studies, thrombosis in mesenteric arterioles and venules was assessed. An abdominal median incision was made, the mesentery was exteriorized, and the artery and/or vein from the second level arch from the bowel were treated with $\mathrm{FeCl}_{3}$ as described above after injection of rhodamine $6 \mathrm{G}$ through the jugular vein. Time to thrombosis was assessed as in the carotid model using intravital microscopy.

After induction of thrombus, the carotid arteries containing thrombi were excised and embedded vertically in OCT (Tissue-Tek; Sakura Finetek) with a proximal-top and distal-bottom position. Serial cross sections (4-6 $\mu \mathrm{m})$ were cut from top to bottom, and sections in the middle portion of the thrombi were used for histological examination. Double immunofluorescence staining was performed using antibodies to CD105 (Ancell), an EC marker, and CD61 (Chemicon), a platelet marker. Nuclei were stained with DAPI. Laser confocal images from 3 serial sections in each animal were taken, and the mean fluorescence intensity (expressed as arbitrary units $/ \mathrm{mm}^{2}$ ) of CD105 staining was determined using NIH ImageJ software (rsb.info.nih.gov/ij/) after converting fluorescence to grayscale.

Human subjects. Studies involving isolation and characterization of MPs from normal human volunteer subjects were approved by the Institutional Review Board of University Hospitals of Cleveland. All other experiments with normal volunteer human subjects - including isolation of blood, platelets, and monocytes - were approved by the Cleveland Clinic Foundation Institutional Review Board. Informed consent was obtained from all subjects. Unless otherwise specified, each experiment was performed with human blood from at least 3 unrelated donors.

Generation and isolation of MPs. Human ECs were isolated from umbilical veins and maintained in culture as described by Jaffe et al. (60). Cells of second passage were typically used for experiments and were incubated with $100 \mathrm{ng} / \mathrm{ml} \mathrm{TNF- \alpha} \mathrm{(R \& D} \mathrm{Systems)} \mathrm{and} 50 \mu \mathrm{g} / \mathrm{ml}$ cycloheximide (Sigma-Aldrich) for 24 hours to generate EMPs. Culture supernatants were collected, and nonviable cells and large cell fragments were removed by centrifugation at $4,300 \mathrm{~g}$ for $5 \mathrm{~min}$. The supernatants were then centrifuged at $100,000 \mathrm{~g}$ for $90 \mathrm{~min}$ at $10^{\circ} \mathrm{C}$ to pellet EMPs. Pelleted EMPs were resuspended in $100 \mu \mathrm{l}$ HEPES-Tyrode buffer $(137 \mathrm{mmol} / \mathrm{l} \mathrm{NaCl}$, $2.8 \mathrm{mmol} / 1 \mathrm{KCl}, 1.0 \mathrm{mmol} / 1 \mathrm{MgCl}_{2}, 12 \mathrm{mmol} / \mathrm{NaHCO}_{3}, 0.4 \mathrm{mmol} / \mathrm{l}$ $\mathrm{Na}_{2} \mathrm{HPO}_{4}, 0.35 \%$ BSA, $10 \mathrm{mmol} / 1$ HEPES, $5.5 \mathrm{mmol} / 1$ glucose, $\mathrm{pH} 7.4$ ) and stored at $-70^{\circ} \mathrm{C}$. Freezing and thawing had no adverse effect on size or morphology of the EMPs, as assessed by immunofluorescence microscopy. EMPs were characterized by flow cytometry using a PCA Analyzer (GUAVA Technologies) and shown to bind FITC-conjugated annexin V (BD Biosciences) and PE-conjugated antibodies to CD105/endoglin (Ancell), VE-cadherin (BD Biosciences), and CD31 (BD Biosciences). In all cases, corresponding isotype-matched nonimmune IgGs were used as controls. CD $105^{+}$EMPs were counted by flow cytometry using 0.3 - and $3-\mu \mathrm{m}$ latex beads (Sigma-Aldrich) as size standards. Each confluent T75 flask of cells generated approximately $3 \times 10^{5} \mathrm{CD}_{105^{+}}$EMPs.
MMPs were derived from human peripheral blood monocytes by stimulation with $12 \mu \mathrm{M} \mathrm{A} 23187$ according to a previously published protocol (61). These were characterized by anti-CD14 and annexin V staining. PMPs were derived from platelets after activation with $1 \mu \mathrm{m} \mathrm{A} 23187$ or thrombin $(0.1 \mathrm{U} / \mathrm{ml})$ according to a previously published protocol (62) and characterized by anti-CD41 and annexin $\mathrm{V}$ staining.

MPs from human blood were isolated from healthy normal subjects. Blood was centrifuged at $500 \mathrm{~g}$ to obtain platelet-poor plasma (PPP). The PPP was then centrifuged at $15,000 \mathrm{~g}$ for 45 minutes at $20^{\circ} \mathrm{C}$ to pellet the MPs, which were resuspended in modified Tyrode buffer containing $0.35 \%$ $\mathrm{BSA}$ and stored at $-70^{\circ} \mathrm{C}$. Human MPs were analyzed by flow cytometry and quantified by light scatter and annexin V staining. MPs were also characterized by cell of origin using antibodies to endothelial (CD105 and CD144), platelet (CD41), and monocytic (CD14) markers. Each marker was separately counted by flow cytometry. Thirteen subjects were analyzed and used for experiments (Table 1).

Preparation of platelets and PRP. Whole blood from healthy human volunteers ranging 24-32 years of age was collected in $0.109 \mathrm{M}$ sodium citrate, $7.4 \mathrm{pH}$ (1:9 dilution). Donors had not taken any medications, including aspirin or NSAIDs, for at least 1 week prior to phlebotomy. PRP was obtained by centrifugation at $100 \mathrm{~g}$ for 12 minutes at room temperature. Platelets were counted in a Z2 particle counter (Coulter), and platelet number was adjusted to $2 \times 10^{8} / \mathrm{ml}$ for all aggregometry experiments. For flow cytometry experiments, washed platelets were prepared by centrifuging $\mathrm{PRP}$ at $600 \mathrm{~g}$ for 10 minutes in the presence of $10 \mathrm{mM}$ prostaglandin $\mathrm{E}_{1}$. The pellet was washed twice with modified Tyrode buffer and then resuspended in this buffer at a concentration of $1 \times 10^{6}$ platelets $/ \mathrm{ml}$.

Detection of platelet CD36 expression. PRP from healthy normal donors $(100 \mu \mathrm{l})$ was incubated with either PE-conjugated anti-CD36 monoclonal antibody (Santa Cruz Biotechnology Inc.) or isotype-matched control antibody. CD36 expression was determined by flow cytometry by gating platelets with an anti-CD42b monoclonal antibody (BD Biosciences) and also on forward versus side scatter, followed by gating on PE fluorescence versus forward scatter for $\mathrm{CD} 42 \mathrm{~b}^{+} \mathrm{CD} 36^{+}$events.

Immunofluorescence flow cytometry detection of platelet-EMP interactions. Washed platelets were incubated with EMPs or with human blood-derived MPs in a ratio of 1:9 for 30 min prior to incubation with the EC-specific anti-CD105 antibody. After antibody incubation, cells were pelleted, washed, and analyzed by flow cytometry. Relative fluorescence intensity histograms and dot plots were made and analyzed with Flow Jo software (version 7.2.4; Tree Star Inc.), and the change in platelet-acquired antiCD105 fluorescence was measured and statistically quantified. In some studies, platelets, EMPs or human blood-derived MPs were incubated with antibodies, oxLDL, annexin $\mathrm{V}$ (BioVision), or monoclonal anti-PS antibody (generously provided by N. Rote, Case Western Reserve University School of Medicine) or its IgM control prior to CD105 staining. Preparation of oxLDL was as previously described (63). Blood collection, EMP or human blood-derived MP incubation, antibody incubation, and data acquisition for all samples were done on the same day, using the same instrument for each individual experiment for consistency.

Immunofluorescence microscopy. For immunofluorescence microscopy, platelets and MPs were labeled with a green fluorophore (Calcein; Invitrogen) and a red fluorophore (PKH-26; Sigma-Aldrich), respectively, and incubated together as described above prior to imaging with a Leica DM-RXE microscope, interfaced to a PC using Q capture software (Quantitative Imaging Company).

Platelet activation studies. Washed platelets $\left(1 \times 10^{6}\right)$ were incubated with EMPs at a 9:1 EMP/platelet ratio or buffer control for 30 minutes and then stimulated with ADP. In studies using human blood-derived MPs, a 9:1 $\mathrm{CD}_{105} \mathrm{MP} /$ platelet ratio was used. For MMPs or PMPs, the platelet/MP 
ratio ranged between 1:10 and 1:40. Platelet-MP mixtures were then incubated for 15 minutes with PE-conjugated anti-P-selectin IgG (BD Biosciences) or FITC-conjugated PAC1 (BD Biosciences), a monoclonal antibody that recognizes the activated conformation of the platelet integrin $\alpha_{2 b} \beta_{3}$. After incubation, the platelets were centrifuged at $700 \mathrm{~g}$ for 10 minutes, resuspended in PBS, and analyzed by flow cytometry. In some studies, washed platelets were preincubated with anti-human CD36 antibody (clone FA6; Invitrogen) or isotype-matched control IgG (Sigma-Aldrich).

Platelet aggregation studies. Platelet aggregation was assessed turbidimetrically with a dual channel aggregometer (Chrono-log Corp.) using graded doses of ADP from 1 to $20 \mu \mathrm{M}$ under constant stirring conditions. The light transmission of PPP was regarded as $100 \%$ aggregation, and the light transmission of PRP before addition of agonist was regarded as $0 \%$. Any change in light transmission after addition of agonist was recorded and expressed as a percentage of deflection.

Statistics. Univariate analyses were performed using ANOVA and paired and unpaired Student's $t$ tests as appropriate. Data are presented as mean \pm SD. A $P$ value less than 0.05 was considered significant. Sta-

1. Clemetson, K.J., Pfueller, S.L., Luscher, E.F., and Jenkins, C.S. 1977. Isolation of the membrane glycoproteins of human blood platelets by lectin affinity chromatography. Biochim. Biophys. Acta. 464:493-508.

2. Rhinehart-Jones, T., and Greenwalt, D.E. 1996. A detergent-sensitive 113-kDa conformer/complex of CD36 exists on the platelet surface. Arch. Biochem. Biophys. 326:115-118.

3. Albert, M.L., et al. 1998. Immature dendritic cells phagocytose apoptotic cells via alphavbeta5 and CD36, and cross-present antigens to cytotoxic T lymphocytes. J. Exp. Med. 188:1359-1368.

4. Knowles, D.M., 2nd, et al. 1984. Monoclonal antihuman monocyte antibodies OKM1 and OKM5 possess distinctive tissue distributions including differential reactivity with vascular endothelium. J. Immunol. 132:2170-2173.

5. Abumrad, N.A., el-Maghrabi, M.R., Amri, E.Z., Lopez, E., and Grimaldi, P.A. 1993. Cloning of a rat adipocyte membrane protein implicated in binding or transport of long-chain fatty acids that is induced during preadipocyte differentiation. Homology with human CD36. J. Biol. Chem. 268:17665-17668.

6. Greenwalt, D.E., and Mather, I.H. 1985. Characterization of an apically derived epithelial membrane glycoprotein from bovine milk, which is expressed in capillary endothelia in diverse tissues. J. Cell Biol. 100:397-408.

7. Greenwalt, D.E., Scheck, S.H., and Rhinehart-Jones, T. 1995. Heart CD36 expression is increased in murine models of diabetes and in mice fed a high fat diet. J. Clin. Invest. 96:1382-1388.

8. Susztak, K., Ciccone, E., McCue, P., Sharma, K., and Bottinger, E.P. 2005. Multiple metabolic hits converge on CD36 as novel mediator of tubular epithelial apoptosis in diabetic nephropathy. PLoS Med. 2:e45.

9. Asch, A.S., Barnwell, J., Silverstein, R.L., and Nachman, R.L. 1987. Isolation of the thrombospondin membrane receptor. J. Clin. Invest. 79:1054-1061.

10. Simantov, R., Febbraio, M., and Silverstein, R.L. 2005. The antiangiogenic effect of thrombospondin-2 is mediated by CD36 and modulated by histidine-rich glycoprotein. Matrix Biol. 24:27-34

11. Endemann, G., et al. 1993. CD36 is a receptor for oxidized low density lipoprotein. J. Biol. Chem. 268:11811-11816

12. Husemann, J., et al. 2002. Scavenger receptors in neurobiology and neuropathology: their role on microglia and other cells of the nervous system. Glia. 40:195-205.

13. Ryeom, S.W., Sparrow, J.R., and Silverstein, R.L.
1996. CD36 participates in the phagocytosis of rod outer segments by retinal pigment epithelium. J. Cell Sci. 109:387-395.

14. Moore, K.J., et al. 2002. A CD36-initiated signaling cascade mediates inflammatory effects of betaamyloid. J. Biol. Chem. 277:47373-47379.

15. Ren, Y., Silverstein, R.L., Allen, J., and Savill, J. 1995. CD36 gene transfer confers capacity for phagocytosis of cells undergoing apoptosis. J. Exp. Med. 181:1857-1862.

16. Stuart, L.M., et al. 2005. Response to Staphylococcus aureus requires CD36-mediated phagocytosis triggered by the $\mathrm{COOH}$-terminal cytoplasmic domain. J. Cell Biol. 170:477-485.

17. Febbraio, M., et al. 2000. Targeted disruption of the class B scavenger receptor CD36 protects against atherosclerotic lesion development in mice. J. Clin. Invest. 105:1049-1056.

18. Jimenez, B., et al. 2000. Signals leading to apoptosis-dependent inhibition of neovascularization by thrombospondin-1. Nat. Med. 6:41-48.

19. Febbraio, M., et al. 1999. A null mutation in murine CD36 reveals an important role in fatty acid and lipoprotein metabolism. J. Biol. Chem. 274:19055-19062.

20. Febbraio, M., Hajjar, D.P., and Silverstein, R.L. 2001. CD36: a class B scavenger receptor involved in angiogenesis, atherosclerosis, inflammation, and lipid metabolism. J. Clin. Invest. 108:785-791.

21. Aitman, T.J., et al. 2000. Malaria susceptibility and CD36 mutation. Nature. 405:1015-1016.

22. Hoebe, K., et al. 2005. CD36 is a sensor of diacylglycerides. Nature. 433:523-527.

23. Laugerette, F., et al. 2005. CD36 involvement in orosensory detection of dietary lipids, spontaneous fat preference, and digestive secretions. J. Clin. Invest. 115:3177-3184.

24. Lin, M., Shieh, S.H., and Yang, T.F. 1993. Frequency of platelet-specific antigens among Chinese in Taiwan. Transfusion. 33:155-157.

25. Seo, D.H., et al. 1998. Gene frequencies of eight human platelet-specific antigens in Koreans. Transfus. Med. 8:129-132.

26. Tomiyama, Y., et al. 1990. Identification of the platelet-specific alloantigen, Naka, on platelet membrane glycoprotein IV. Blood. 75:684-687.

27. Urwijitaroon, Y., Barusrux, S., Romphruk, A., and Puapairoj, C. 1995. Frequency of human platelet antigens among blood donors in northeastern Thailand. Transfusion. 35:868-870.

28. Podrez, E.A., et al. 2007. Platelet CD36 links hyperlipidemia, oxidant stress and a prothrombotic phenotype. Nat. Med. 13:1086-1095.

29. Fadok, V.A., et al. 1998. The role of phosphatidylserine in recognition of apoptotic cells by phago- cytes. Cell Death Differ. 5:551-562.
0. Greenberg, M.E., et al. 2006. Oxidized phosphatidylserine-CD36 interactions play an essential role in macrophage-dependent phagocytosis of apoptotic cells. J. Exp. Med. 203:2613-2625.

31. Pittoni, V., and Valesini, G. 2002. The clearance of apoptotic cells: implications for autoimmunity. Autoimmun. Rev. 1:154-161.

32. Combes, V., et al. 1999. In vitro generation of endothelial microparticles and possible prothrombotic activity in patients with lupus anticoagulant. J. Clin. Invest. 104:93-102.

33. Jimenez, J.J., et al. 2003. Endothelial cells release phenotypically and quantitatively distinct microparticles in activation and apoptosis. Thromb. Res. 109:175-180.

34. Falati, S., et al. 2003. Accumulation of tissue factor into developing thrombi in vivo is dependent upon microparticle P-selectin glycoprotein ligand 1 and platelet P-selectin. J. Exp. Med. 197:1585-1598.

35. Sims, P.J., Wiedmer, T., Esmon, C.T., Weiss, H.J., and Shattil, S.J. 1989. Assembly of the platelet prothrombinase complex is linked to vesiculation of the platelet plasma membrane. Studies in Scott syndrome: an isolated defect in platelet procoagulant activity. J. Biol. Chem. 264:17049-17057.

36. Sabatier, F., et al. 2002. Interaction of endothelial microparticles with monocytic cells in vitro induces tissue factor-dependent procoagulant activity. Blood. 99:3962-3970.

37. Bernal-Mizrachi, L., et al. 2003. High levels of circulating endothelial microparticles in patients with acute coronary syndromes. Am. Heart J. 145:962-970.

38. Shet, A.S., et al. 2003. Sickle blood contains tissue factor-positive microparticles derived from endothelial cells and monocytes. Blood. 102:2678-2683.

39. Davi, G., and Ferroni, P. 2005. Microparticles in type 2 diabetes mellitus. J. Thromb. Haemost. 3:1166-1167.

40. Koga, H., et al. 2005. Elevated levels of VE-cadherinpositive endothelial microparticles in patients with type 2 diabetes mellitus and coronary artery disease. J. Am. Coll. Cardiol. 45:1622-1630.

41. Jimenez, J.J., et al. 2001. Elevated endothelial microparticles in thrombotic thrombocytopenic purpura: findings from brain and renal microvascular cell culture and patients with active disease. Br. J. Haematol. 112:81-90.

42. Dignat-George, F., et al. 2004. Endothelial microparticles: a potential contribution to the thrombotic complications of the antiphospholipid syndrome. Thromb. Haemost. 91:667-673.

43. Gonzalez-Quintero, V.H., et al. 2004. Elevated plasma endothelial microparticles: preeclampsia versus gestational hypertension. Am.J. Obstet. Gyne- 
col. 191:1418-1424.

44. Pihusch, V., et al. 2006. Endothelial cell-derived microparticles in allogeneic hematopoietic stem cell recipients. Transplantation. 81:1405-1409.

45. Berckmans, R.J., et al. 2001. Cell-derived microparticles circulate in healthy humans and support low grade thrombin generation. Thromb. Haemost. 85:639-646

46. Simák, J., Holada, K., D’Agnillo, F., Janota, J., and Vostal, J.G. 2002. Cellular prion protein is expressed on endothelial cells and is released during apoptosis on membrane microparticles found in human plasma. Transfusion. 42:334-342.

47. Brogan, P.A., et al. 2004. Endothelial and platelet microparticles in vasculitis of the young. Artbritis Rheum. 50:927-936.

48. Simak, J., and Gelderman, M.P. 2006. Cell membrane microparticles in blood and blood products: potentially pathogenic agents and diagnostic markers. Transfus. Med. Rev. 20:1-26.

49. Rahaman, S.O., et al. 2006. A CD36-dependent signaling cascade is necessary for macrophage foam cell formation. Cell Metab. 4:211-221.

50. Prevost, N., et al. 2005. Eph kinases and ephrins support thrombus growth and stability by regulat- ing integrin outside-in signaling in platelets. Proc. Natl. Acad. Sci. U. S. A. 102:9820-9825.

51. Prevost, N., et al. 2004. Signaling by ephrinB1 and Eph kinases in platelets promotes Rap1 activation, platelet adhesion, and aggregation via effector pathways that do not require phosphorylation of ephrinB1. Blood. 103:1348-1355.

52. Angelillo-Scherrer, A., et al. 2005. Role of Gas6 receptors in platelet signaling during thrombus stabilization and implications for antithrombotic therapy. J. Clin. Invest. 115:237-246.

53. Angelillo-Scherrer, A., et al. 2001. Deficiency or inhibition of Gas 6 causes platelet dysfunction and protects mice against thrombosis. Nat. Med. 7:215-221.

54. Falati, S., et al. 2006. Platelet PECAM-1 inhibits thrombus formation in vivo. Blood. 107:535-541.

55. Andre, P., et al. 2002. CD40L stabilizes arterial thrombi by a beta3 integrin-dependent mechanism. Nat. Med. 8:247-252.

56. Henn, V., Steinbach, S., Buchner, K., Presek, P., and Kroczek, R.A. 2001. The inflammatory action of CD40 ligand (CD154) expressed on activated human platelets is temporally limited by coexpressed CD40. Blood. 98:1047-1054.

57. Zhu, L., et al. 2007. Regulated surface expression and shedding support a dual role for semaphorin $4 \mathrm{D}$ in platelet responses to vascular injury. Proc. Natl. Acad. Sci. U. S. A. 104:1621-1626.

58. Brass, L.F., Jiang, H., Wu, J., Stalker, T.J., and Zhu, L. 2006. Contact-dependent signaling events that promote thrombus formation. Blood Cells Mol. Dis. 36:157-161.

59. Brass, L.F., Zhu, L., and Stalker, T.J. 2005. Minding the gaps to promote thrombus growth and stability. J. Clin. Invest. 115:3385-3392.

60. Jaffe, E.A., et al. 1989. IFN-gamma and IFN-alpha induce the expression and synthesis of Leu 13 antigen by cultured human endothelial cells. J. Immunol. 143:3961-3966.

61. Cerri, C., et al. 2006. Monocyte/macrophagederived microparticles up-regulate inflammatory mediator synthesis by human airway epithelial cells. J. Immunol. 177:1975-1980.

62. Barry, O.P., Pratico, D., Lawson, J.A., and FitzGerald, G.A. 1997. Transcellular activation of platelets and endothelial cells by bioactive lipids in platelet microparticles. J. Clin. Invest. 99:2118-2127.

63. Morel, D.W., and Chisolm, G.M. 1989. Antioxidant treatment of diabetic rats inhibits lipoprotein oxidation and cytotoxicity. J. Lipid Res. 30:1827-1834. 